$$
\text { M960 } 050367
$$

\title{
A Comparison of Numerical Schemes for Transport Problems
}

\author{
Larry Carson \\ James M. Ferguson
}

\section{FREIVED \\ SEP 09 1996 \\ OSTI}

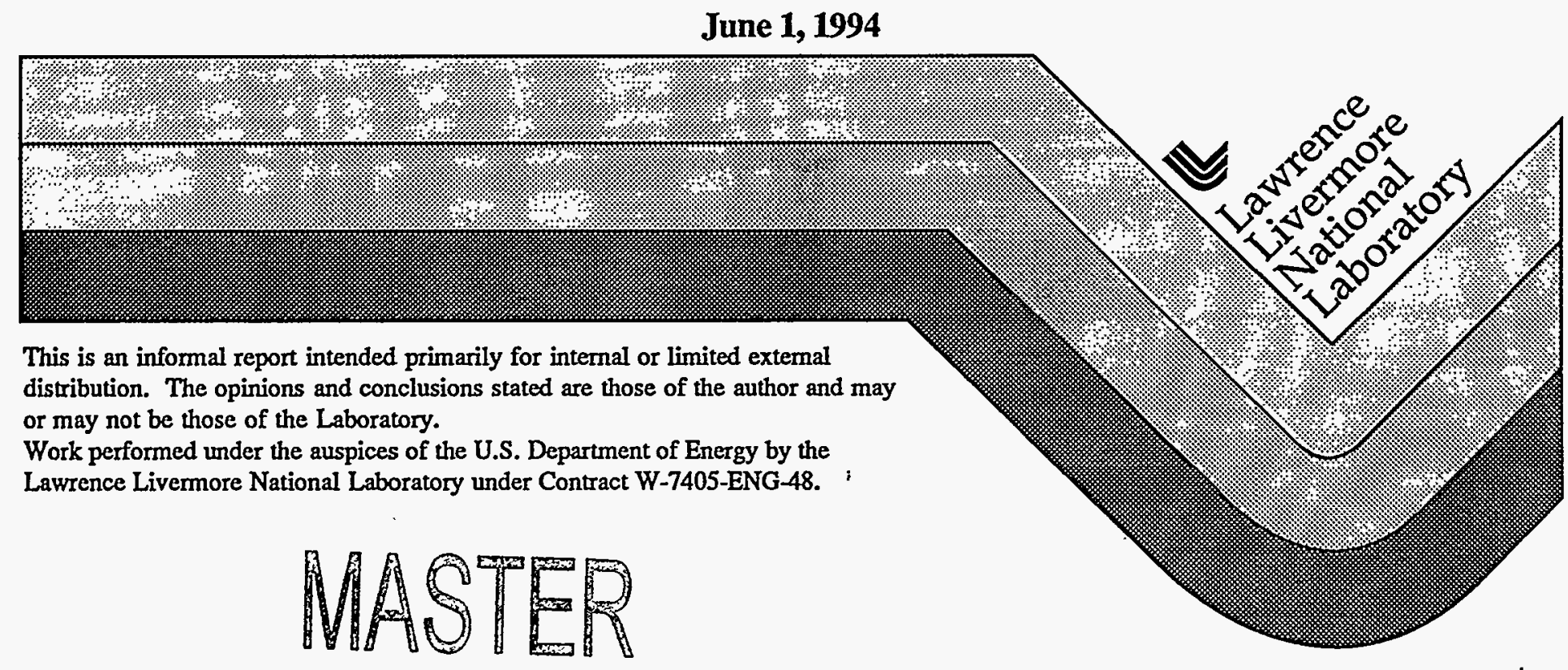


This document was prepared as an account of work sponsored by an agency of the United States Government. Neither the United States Government nor the University of California nor any of their employees, makes any warranty, express or implied, or assumes any legal liability or responsibility for the accuracy, completeness, or usefulness of any information, apparatus, product, or process disclosed, or represents that its use would not infringe privately owned rights. Reference herein to any specific commercial product, process, or service by trade name, trademark, manufacturer, or otherwise, does not necessarily constitute or imply its endorsement, recommendation, or favoring by the United States Government or the University of California. The views and opinions of authors expressed herein do not necessarily state or reflect those of the United States Government or the University of California, and shall not be used for advertising or product endorsement purposes.

This report has been reproduced directly from the best available copy.

Available to DOE and DOE contractors from the

Office of Scientific and Technical Information

P.O. Box 62, Oak Ridge, TN 37831

Prices available from (615) 576-8401, FTS 626-8401

Available to the public from the

National Technical Information Service

U.S. Department of Commerce

5285 Port Royal Rd.,

Springfield, VA 22161 


\section{DISCLAIMER}

Portions of this document may be illegible in electronic image products. Images are produced from the best available original document. 


\title{
Lawrence Livermore National Laboratory
}

\section{A Comparison of Numerical Schemes for Transport Problems ${ }^{1}$}

Larry Carson and James M. Ferguson

Lawrence Livermore National Laboratory

P.O. Box 808, L-35, Livermore, CA 94550

\begin{abstract}
Five finite-difference schemes for solving the time-independent, singlegroup transport equation in planar geometry.are compared for accuracy and computational speed. We examine in detail two cases: one in which the medium is purely absorbing and another in which isotropic scattering and uniformly distributed sources are also present. In both cases, we find that among the schemes examined, the one based on linear and quadratic finite elements achieves superior accuracy at a given computational cost whenever the zone width is less than a mean free path.
\end{abstract}

Mathematics Subject Classifications: 65M05, 65M60, 82A70 


\section{Introduction}

Over the last few years, significant advances have been made in the deterministic solution of the linear Boltzmann equation [1] describing the transport of particles through an absorbing and scattering medium. These advances concern the finite difference scheme that is applied to the transport equation, whose solution gives the flux $\psi(\mathbf{x}, E, \Omega)$ of particles of energy $E$ flowing in direction $\Omega$ at the point $\mathbf{x}$.

Previously, the scheme of choice was the diamond difference (DD) scheme [2,3] since it is relatively simple to implement, is second-order accurate in the spatial zone width, and possesses the correct diffusion limit [4]. However, it was recently discovered that, if optically thick zoning is employed, the DD scheme breaks down when sharp boundaries are present in the problem [5]. Besides failing to adequately account for the (unresolved) boundary layer, the DD flux penetrating a sharp interface acquires an unphysical, oscillating component that may lead to negative fluxes.

To deal with these difficulties, a class of finite difference schemes based on the linear discontinuous finite element method have been developed [5-9]. Like the DD scheme, these schemes are in general second-order accurate and, at least in problems respecting planar symmetry (e.g. slab geometry), have been shown to possess the proper diffusion limit. At the cost of somewhat greater complexity, the linear discontinuous schemes also treat sharp interfaces quite accurately. One such scheme (which we label the MLD scheme below) has been singled out for its particular robustness.

This work is concerned with extending these investigations to other schemes as complex as the linear discontinuous schemes but which are potentially more accurate. One is the Galerkin finite element method which approximates functions by linear, piecewise-continuous elements and computes residuals of the transport equation with respect to the same set of functions [10]. The other is a scheme approximating functions by linear combinations of linear and quadratic piecewise-continuous finite elements. Applied to the simple case of the free-space, one-dimensional transport equation, $\psi_{, t}-\psi_{, x}=0$, one may show that both of these schemes produce solutions that are fourth-order accurate. This naturally leads us to inquire whether these schemes can generate highly accurate solutions when absorption and scattering terms are also present.

Using on a combination of analysis and numerical experiments, we shall apply five finite difference schemes to the linear Boltzmann equation: the usual diamond difference scheme, two schemes based on linear discontinuous elements, and the Galerkin 
and quadratic schemes. We shall restrict our attention to the single-group linear transport equation in slab geometry with isotropic scattering, given by [1]

$$
\mu \frac{d \psi(z, \mu)}{d z}+\sigma_{T}(z) \psi(z, \mu)=\left(\sigma_{T}(z)-\sigma_{A}(z)\right) \int_{-1}^{1} d \mu \psi(z, \mu)+Q(z)
$$

where $\mu=\hat{\mathbf{z}} \cdot \Omega$ is the projection of the flux direction along the $z$ axis, $\sigma_{T}(z)$ and $\sigma_{A}(z)$ are the total and absorption cross sections and $Q(z)$ is an isotropic source of flux.

Similar surveys of finite difference schemes applied to the plane-symmetric transport equation (1) have appeared in the literature [11-16]. In many of these studies, schemes are developed using the method of characteristics [17] to recast the transport equation over a spatial zone $[z, z+\Delta z]$ in the integral form,

$$
\psi(z+\Delta z, \mu)-\psi(z, \mu)=\int_{z}^{z+\Delta z} d z^{\prime} G\left(z-z^{\prime}, \mu\right) S\left(z^{\prime}\right)
$$

where the kernel $G$ is approximated by

$$
G\left(z-z^{\prime}, \mu\right) \simeq \exp \left[-\sigma_{T}\left(\frac{z+z^{\prime}}{2}\right) \frac{z-z^{\prime}}{\mu}\right]
$$

and $S(z)$ is the right-hand-side of Eq. (1). (In Eq. (3), the total cross section $\sigma_{T}(z)$ is assumed to be constant over the width of the zone.) The source function $S\left(z^{\prime}\right)$ is then expanded in polynomials of varying orders in $z^{\prime}$ to derive the schemes of interest. While this procedure oftentimes leads to schemes of high accuracy, it is not readily generalizable to higher dimensions or more complex geometries. Besides the complications involved in constructing the appropriate kernel $G\left(z-z^{\prime}, \Omega\right)$, it is problematic to select a representative flux $\psi^{\text {out }}$ on the outflow boundary of a zone in more than one spatial dimension. An improper choice of $\psi^{\text {out }}$ often results in solutions of poor accuracy (see, for example, [18]).

Therefore, in seeking to obtain results that may generalize to higher dimensions, our approach is to solve the transport equation in its differential form (1). With regard to the accuracy of the diamond difference and linear discontinuous schemes, our results agree with the surveys of Alcouffe et al. [12], Lee and Vaidyanathan [13], and Larsen and Miller [14]. Martin and Duderstadt have examined quadratic finite elements in both space and angle in Ref. [11], achieving results that are qualitatively similar to our own. Ferguson [19] has also applied quadratic elements to the case of spherical geometry, obtaining results superior to the diamond-difference scheme and providing much of the motivation for our present investigation. 
In section 2, we begin with the simplest case of a purely absorbing medium where $\sigma_{T}=\sigma_{A}$ is constant and there is no source term $(Q=0)$. Since the flux direction $\mu$ appears only as a parameter in the transport equation, full analytic solutions of both the continuum and discrete equations are easily obtained and compared. Besides discerning the accuracy of each scheme, we shall also determine how this accuracy may be degraded in the actual solution by a poor choice of starting conditions. As a byproduct, we also extract conditions on the zone width $h$ that must be imposed if we wish to eschew negative fluxes. In section 3, the case of uniform scattering cross sections $\sigma_{S}=\sigma_{T}-\sigma_{A} \neq 0$ and sources $Q$ is taken up. The angular variable $\mu$ is discretized using familiar Gauss-Legendre quadratures. Analytic and numerical solutions of the continuum and discrete equations are obtained in the case of two ordinates, with qualitative results that are confirmed with numerical computations using 8 ordinates. Section 4 is reserved for a summary and concluding remarks.

\section{Transport through a purely absorbing medium}

Taking $Q=0$ and $\sigma_{T}=\sigma_{A}=$ constant in Eq. (1) and rescaling the coordinate $z$ via $x \equiv \sigma_{T} z / \mu$, we obtain

$$
\frac{d \psi(x)}{d x}+\psi(x)=0
$$

describing transport through a sourceless, purely absorbing medium (we have dropped all explicit reference to the flux angle $\mu$ ). If we know the flux at any point, say $x_{0}=0$, then the solution of (4) is given by

$$
\psi^{\text {cont }}(x)=\psi(0) e^{-x},
$$

where the notation "cont" denotes "continuum".

We shall formulate the various difference schemes for numerically solving this equation using the Petrov-Galerkin finite element method. (For an excellent discussion of the finite element method applied to the transport equation, see Ref. [11].) To wit, let us define the inner product,

$$
\langle f, g\rangle=\int_{-\infty}^{\infty} d x f(x) g(x)
$$

and establish a grid of discrete points $\left\{x_{j}\right\}$ for the coordinate $x$ (for concreteness, we take $x_{0}=0$ ). The flux density $\psi(x)$ is expanded in a set of trial functions $u_{j}(x)$ which have support in some finite range of $x$, eg. $\left[x_{j-1}, x_{j}\right]$ or $\left[x_{j-1}, x_{j+1}\right]$. The finite 
difference equations are then determined by requiring that the residuals of Eq. (4) with respect to a set of test functions $v_{j}(x)$ vanish:

$$
\left\langle v_{j}, \frac{d \psi}{d x}+\psi\right\rangle=0
$$

To be a well-defined procedure, the cardinality of the trial functions $u_{j}$ must match that of the test functions $v_{j}$.

As first shown in Ref. 20, the well-known diamond difference scheme can be cast in Petrov-Galerkin form. For this we take as trial functions the 'teepee' functions,

$$
\varphi_{j}(x)= \begin{cases}\frac{x-x_{j-1}}{\Delta x_{j}}, & x_{j-1} \leq x \leq x_{j} \\ \frac{x_{j+1}-x}{\Delta x_{j+1}}, & x_{j} \leq x \leq x_{j+1} \\ 0, & \text { otherwise }\end{cases}
$$

where $\Delta x_{j} \equiv x_{j}-x_{j-1}$, while for the test functions we take the histogram functions,

$$
H_{j}(x)= \begin{cases}1, & x_{j-1} \leq x \leq x_{j} \\ 0, & \text { otherwise }\end{cases}
$$

Expanding $\psi(x)$ via

$$
\psi(x)=\sum_{j} \psi_{j} \varphi_{j}(x)
$$

with constant coefficients $\psi_{j}$, one obtains

$$
\left\langle H_{j}, \frac{d \psi}{d x}+\psi\right\rangle=\psi_{j}-\psi_{j-1}+\frac{\Delta x_{j}}{2}\left(\psi_{j}+\psi_{j-1}\right)=0
$$

The values of the coefficients $\psi_{j}, j \neq 0$, are determined by (8) given the initial value $\psi_{0}=\psi(0)$.

The linear discontinuous scheme results from expanding $\psi(x)$ in piecewise linear. (but not necessarily continuous) elements in every cell $\left[x_{j-1}, x_{j}\right]$. For this we use as trial functions the histogram functions defined above and the slope functions defined by

$$
\tilde{H}_{j}(x)= \begin{cases}\frac{x-x_{j-1 / 2}}{\Delta x_{j} / 2}, & x_{j-1} \leq x \leq x_{j} \\ 0, & \text { otherwise }\end{cases}
$$

where $x_{j-1 / 2}=\left(x_{j}+x_{j-1}\right) / 2$. Expanding

$$
\psi(x)=\sum_{j}\left(\psi_{j} H_{j}(x)+\tilde{\psi}_{j} \tilde{H}_{j}(x)\right)
$$


we see that $\psi_{j}, \psi_{j}+\tilde{\psi}_{j}$ and $\psi_{j}-\tilde{\psi}_{j}$ give the centered, right-edge and left-edge fluxes in the $j$ th cell, respectively. The desired difference equations are then obtained using as test functions $v_{j}$ the same set $\left\{H_{j}, \tilde{H}_{j}\right\}$, with the following important modification: Instead of Eq. (6), we impose

$$
0=\left\langle v_{j}, \frac{d \psi}{d x}+\psi\right\rangle+v_{j}\left(x_{j-1}^{+}\right)\left[\psi\left(x_{j-1}^{+}\right)-\psi\left(x_{j-1}^{-}\right)\right]
$$

$\left(\psi\left(x^{ \pm}\right)=\lim _{\epsilon \rightarrow 0} \psi(x \pm \epsilon)\right)$, where the additional term enforces in weak form the inflow boundary condition on the $j$ th cell. The final result is, for each $j$, the $2 \times 2$ matrix difference equation,

$$
\left[\Delta x_{j}\left(\begin{array}{cc}
1 & 0 \\
0 & \theta
\end{array}\right)+\left(\begin{array}{cc}
1 & 1 \\
-1 & 1
\end{array}\right)\right]\left(\begin{array}{c}
\psi_{j} \\
\tilde{\psi}_{j}
\end{array}\right)+\left(\psi_{j-1}+\tilde{\psi}_{j-1}\right)\left(\begin{array}{c}
-1 \\
1
\end{array}\right)=0
$$

with $\theta=1 / 3$. Given an initial value for $\psi_{0}+\tilde{\psi}_{0}$, this equation can be used to determine $\psi_{j}$ and $\tilde{\psi}_{j}$ for any value $j \neq 0$. Thus, like the DD scheme and the continuum solution (5), only a single datum is required to initialize the scheme.

As shown by Szilard and Pomraning [7], Eq. (10) defines a valid finite difference scheme for any value of $\theta$, and reduces to the standard DD scheme when $\theta=0$. For example, instead of using $H_{j}$ and $\tilde{H}_{j}$ for test functions in Eq. (9), one could use histograms defined on the half-cells, $\left[x_{j-1}, x_{j-1 / 2}\right]$ and $\left[x_{j-1 / 2}, x_{j}\right]$. It is a simple exercise to show that Eq. (10) again results, this time with $\theta=1 / 2$. Thus it is of interest to leave $\theta$ arbitrary in the analysis that follows, and thereby define a class of modified linear discontinuous schemes labelled by $\theta$. As we shall see, the value $\theta=1$ is special; the corresponding scheme is called the 'mass-lumped' linear discontinuous (MLD) scheme in the literature. We shall distinguish the original scheme with $\theta=1 / 3$ by labelling it the LD scheme.

The Galerkin scheme is produced by expanding the flux density in teepee functions as in Eq. (7), but this time we test with the same teepee functions. This leads to the system of equations given by

$$
\begin{aligned}
0 & =\left\langle\varphi_{j}, \frac{d \psi}{d x}+\psi\right\rangle \\
& =\frac{1}{2}\left(\psi_{j+1}-\psi_{j-1}\right)+\frac{\Delta x_{j}}{6} \psi_{j-1}+\frac{1}{3}\left(\Delta x_{j}+\Delta x_{j+1}\right) \psi_{j}+\frac{\Delta x_{j+1}}{6} \psi_{j+1} .
\end{aligned}
$$

Notice that the scheme requires three points in the site index $j$ and so, unlike the DD, LD, and MLD schemes, must be initialized with $\psi_{0}$ and an additional condition on the coefficients $\left\{\psi_{j}\right\}$. We shall return to this point in a moment. 
The last scheme we shall consider expands the flux in linear and quadratic elements, viz.

$$
\psi(x)=\sum_{j}\left(\psi_{j} \varphi_{j}(x)+\tilde{\psi}_{j} \tilde{\varphi}_{j}(x)\right)
$$

where

$$
\tilde{\varphi}_{j}(x)=\left\{\begin{array}{ll}
4 \frac{\left(x_{j}-x\right)\left(x-x_{j-1}\right)}{\left(\Delta x_{j}\right)^{2}}, & x_{j-1} \leq x \leq x_{j}, \\
0, & \text { otherwise, }
\end{array} .\right.
$$

Testing with the histogram and slope functions, $H_{j}(x)$ and $\tilde{H}_{j}(x)$, respectively, we obtain

$$
\begin{aligned}
& \psi_{j}-\psi_{j-1}+\frac{\Delta x_{j}}{2}\left(\psi_{j}+\psi_{j-1}\right)+\frac{\left(\Delta x_{j}\right)^{2}}{12}\left(\psi_{j}-\psi_{j-1}\right)=0 \\
& \tilde{\psi}_{j}=\frac{\Delta x_{j}}{8}\left(\psi_{j}-\psi_{j-1}\right) .
\end{aligned}
$$

We now specialize to the case of uniform intervals: $\Delta x_{j}=h$, for all $j$. In this case, a general solution of Eqs. (8), (10), (11), and (13) may be found by employing the Ansatz,

$$
\psi_{j}=\Psi r^{j},
$$

where $\Psi$ and $r$ are constants to be determined. In the case of the DD scheme, for example, this substitution leads to the auxiliary equation for $r$,

$$
r-1+\frac{h}{2}(r+1)=0,
$$

while $\Psi$ is simply $\psi_{0}$. Hence we have the solution,

$$
\begin{aligned}
r & =r_{+}=\frac{1-h / 2}{1+h / 2}, \\
\psi_{j}^{D D} & =\psi_{0} r_{+}^{j} .
\end{aligned}
$$

The continuum limit of this expression is easily extracted. Taking $j \rightarrow \infty$ and $h \rightarrow 0$ such that the product $j h=x$ is fixed, one finds that

$$
\log r_{+}^{j} \rightarrow-x\left(1+\frac{h^{2}}{12}+\mathcal{O}\left(h^{4}\right)\right),
$$

so that in the same limit,

$$
\psi_{j}^{D D} \rightarrow \psi_{0} e^{-x}\left(1-\frac{x h^{2}}{12}+\mathcal{O}\left(h^{4}\right)\right) .
$$


Thus, taking $\psi_{0}=\psi(0)$, the continuum solution (5) is retrieved, up to corrections of order $h^{2}$, verifying the second-order accuracy of the DD scheme.

The solution (15) of the DD equations is useful in another respect, namely, in determining conditions under which negative fluxes and/or oscillating behaviour could occur. Evidently, both are avoided if $r_{+}>0$ or $h=\sigma_{T} \Delta z / \mu<2$, that is, if the zone width $\Delta z$ is less than twice the projected mean free path $\mu / \sigma_{T}$.

In applying Ansatz (14) to the linear discontinuous schemes, we treat $\Psi$ as a two-component vector and arrive at the equation,

$$
\left(\begin{array}{cc}
-1+(1+h) r & -1+r \\
1-r & 1+(1+\theta h) r
\end{array}\right) \Psi=0 .
$$

This has a nontrivial solution only if the indicated matrix has zero determinant, implying that $r=0$ or $r=r_{+}$, where

$$
r_{+}=\frac{1-(1-\theta) h / 2}{1+(1+\theta) h / 2+\theta h^{2} / 2} \text {. }
$$

The corresponding (unnormalized) eigenvectors are

$$
\Psi^{(0)}=\left(\begin{array}{c}
1 \\
-1
\end{array}\right) \text { and } \Psi^{(t)}=\left(\begin{array}{l}
a \\
b
\end{array}\right)
$$

where

$$
a=\frac{1+\theta h / 2}{1+(1+\theta) h / 2+\theta h^{2} / 2} \text { and } b=-\frac{h / 2}{1+(1+\theta) h / 2+\theta h^{2} / 2} .
$$

Therefore, a general solution to $(10)$ is of the form,

$$
\left(\begin{array}{c}
\psi_{j} \\
\tilde{\psi}_{j}
\end{array}\right)=\alpha_{0} \Psi^{(0)} \delta_{j 0}+\alpha_{+} \Psi^{(+)} r_{+}^{j},
$$

where $\alpha_{0}$ and $\alpha_{+}$are constants to be determined. One way to fix these constants is to specify $\psi_{0}$ and $\tilde{\psi}_{0}$ and invert the relation,

$$
\left(\begin{array}{cc}
1 & a \\
-1 & b
\end{array}\right)\left(\begin{array}{c}
\alpha_{0} \\
\alpha_{+}
\end{array}\right)=\left(\begin{array}{c}
\psi_{0} \\
\tilde{\psi}_{0}
\end{array}\right)
$$

following from (17).

The term proportional to $\Psi^{(+)}$in (17) is evidently the physical solution of interest, while the $\Psi^{(0)}$ term is an artefact of the discretization scheme. In the continuum limit, one finds

$$
r_{+}^{j} \rightarrow e^{-x}\left(1+\left(\theta-\frac{1}{3}\right) \frac{h^{2}}{4} x-\frac{\theta^{2} h^{3}}{8} x+\mathcal{O}\left(h^{4}\right)\right),
$$


so that, provided the constants $\alpha_{0}$ and $\alpha_{+}$are properly adjusted, we may secure second-order accuracy for arbitrary $\theta$ and third-order accuracy if $\theta=1 / 3$. This is easy to arrange by requiring that the right-edge flux $\psi_{0}+\tilde{\psi}_{0}$ impingent on the first cell has the initial value $\psi(0)$. One then finds that, for all $j$,

$$
\psi_{j}^{(M) L D} \equiv \psi_{j}+\tilde{\psi}_{j}=\psi(0) r_{+}^{j} .
$$

These solutions properly converge to the continuum solution (5) as $h \rightarrow 0$. The artefact $\Psi^{(0)}$, present only for $j=0$, merely reflects the one-parameter ambiguity in using two numbers, $\psi_{0}$ and $\tilde{\psi}_{0}$, to specify the incident flux $\psi(0)$.

A necessary condition for avoiding oscillatory behaviour and negative fluxes in the discrete solution (17) is that $r_{+}>0$. From Eq. (16) we see that, for $\theta \neq 1$, this is the case if

$$
h<\frac{2}{1-\theta},
$$

which reduces to $h<3$ for the LD scheme. However, if $\theta=1, r_{+}$is unconditionally positive for all values of $h$ [7], which probably explains the 'robustness' observed by Larsen and Morel [5] when using the MLD scheme.

The auxiliary equation of the Galerkin scheme is

$$
r^{2}-1+\frac{h}{3}\left(r^{2}+4 r+1\right)=0
$$

whose roots $r_{+}$and $-r_{-}$are given by

$$
\left(1+\frac{h}{3}\right) r_{ \pm}=\mp \frac{2 h}{3}+\sqrt{1+\frac{h^{2}}{3}}
$$

Thus the general solution of Eq. (11) is

$$
\psi_{j}=\alpha_{+} r_{+}^{j}+\alpha_{-}(-1)^{j} r_{-}^{j},
$$

where $\alpha_{ \pm}$are constants to be determined by starting conditions. Unlike schemes so far examined, there are not one but two constants specifying a general solution of the Galerkin equations (11). This follows from a mismatch in the number of trial and test functions used to construct the scheme. Since the incoming flux $\psi(0)$ specifies only one condition on the constants, another equation must be found to determine the discrete solution. Another problem is the presence of a component that oscillates for any value of $h$, signalling the probable presence of negative fluxes. 
In fact, the oscillatory term is an unphysical artefact of the scheme, as may confirmed by examining the continuum limits of $r_{+}^{j}$ and $r_{-}^{j}$ :

$$
\begin{aligned}
& r_{+}^{j} \rightarrow \exp (-x)\left(1-\frac{x h^{4}}{180}+\mathcal{O}\left(h^{6}\right)\right) \\
& r_{-}^{j} \rightarrow \exp \left(\frac{x}{3}\right)\left(1-\frac{2 x h^{2}}{81}+\mathcal{O}\left(h^{4}\right)\right) .
\end{aligned}
$$

The factor $r_{+}^{j}$ coincides with the continuum solution up to terms of order $h^{4}$. However, the potential fourth-order accuracy of the total solution is degraded to $\mathcal{O}\left(h^{n}\right)$ with $n<4$ if $\alpha_{-}$is nonzero and of order $h^{n}$. Furthermore, the unphysical component grows exponentially with $x$.

Ideally, we would like to specify $\psi_{0}$ and $\psi_{1}$ so that $\alpha_{+}=\psi(0)$ and $\alpha_{-}=0$. If we take $\psi_{0}=\psi(0)$, then this corresponds to setting

$$
\psi_{1}=r_{+} \psi_{0}=\psi(0)\left(1-h+\frac{h^{2}}{2}-\frac{h^{3}}{3 !}+\frac{h^{4}}{4 !}-\frac{h^{5}}{72}+\mathcal{O}\left(h^{6}\right)\right)
$$

For this choice of $\psi_{0}$ and $\psi_{1}$, full fourth-order accuracy is achieved. Notice, however, that this presumes full knowledge of the general solution (20), specifically the precise expression (19) for $r_{+}$.

Let's see what happens if we apply cruder initializations in the first cell. One natural choice is to take $\psi(x)$ constant in the first cell, corresponding to the choice $\psi_{0}=\psi_{1}=\psi(0)$. One finds that

$$
\alpha_{+} / \psi(0)=1-\alpha_{-} / \psi(0)=1+\frac{h}{2}+\mathcal{O}\left(h^{2}\right)
$$

We see that the nonphysical component is only suppressed to first order in $h$. Similarly, the physical component deviates from its continuum value by a term linear in $h$. However, it is easy to improve upon this initialization. A better choice would relate, for instance, $\psi_{0}$ with $\psi_{1}$ using the DD scheme (8). To first order in $h$, this gives the condition,

$$
\psi_{1}=\psi_{0}(1-h)
$$

and corresponds to the first two terms in the series expansion (21). Together with $\psi_{0}=\psi(0)$, this implies

$$
\alpha_{+} / \psi(0)=1-\alpha_{-} / \psi(0)=1-\frac{h^{2}}{4}+\mathcal{O}\left(h^{4}\right),
$$

and we have secured one more order of accuracy. 
The relation between initialization and accuracy in this simple example is now evident: Taking on successive terms of (21) in the relation between $\psi_{1}$ and $\psi_{0}$, one can produce schemes of progressively higher accuracy. Notice that one must accurately initialize to order $h^{3}$ before the fourth-order accuracy of the scheme is regained. This will clearly be impractical in more complex situations, and presents a dilemma for the useful application of the $G$ scheme. Indeed, in this approach to initialization, the best one can usually do is to start with a one-step method such as the DD scheme, which in the present context corresponds to the starting condition (22) linear in $h$, and leads only to second-order accuracy. Even the subdivision of the first cell into $N$ subcells of width $h / N$, and $N$ repeated applications of the $\mathrm{DD}$ scheme over these subcells, will not increase accuracy beyond second-order, but merely reduce the error by a factor of $1 / N$.

So far, we have discussed initializing the G-scheme with some two-point scheme on the inflow boundary. Let's now examine what happens if, say, the two-point DD scheme is applied to the outflow boundary at $L=N h$ :

$$
0=\psi_{N}-\psi_{N-1}+\frac{h}{2}\left(\psi_{N}+\psi_{N-1}\right) \text {. }
$$

This equation results in the Petrov-Galerkin formalism from testing the $N$ th zone - with the histogram function $H_{N}(x)$. Now, instead of (23), we have the result that, as $h \rightarrow 0$ (but $L=N h$ remains fixed and finite),

$$
\alpha_{+} / \psi(0)=1-\alpha_{-} / \psi(0)=1+(-1)^{N} e^{-4 L / 3}\left(\frac{h^{3}}{24}+\mathcal{O}\left(h^{4}\right)\right)+\mathcal{O}\left(e^{-8 L / 3}\right) .
$$

We observe that the unphysical component $\alpha_{-}$is exponentially suppressed. Thus, to the extent that transport occurs through an optically thick region, allowing us to neglect exponential corrections, it appears that the fourth-order accuracy of the scheme is regained.

Actually, this last statement needs some qualification. Supposing the result (25), then as $h \rightarrow 0$, our solution is of the form (recalling $x \equiv j h$ ),

$$
\psi_{j}^{G}-\psi^{\text {cont }}(x) \rightarrow-\frac{h^{3}}{12}\left(\frac{(-1)^{N+j}}{2} e^{-4 L / 3+x / 3}+\frac{h}{15} x e^{-x}\right) \psi(0)+\ldots
$$

where here and hereafter the ellipsis denotes higher order corrections of relative order $h$ and/or $e^{-L}$. So even if $L>>1$, the two deviations to the continuum solution $\psi^{\text {cont }}(x)$ may be comparable to each other for $x \sim L$. A global measure of the relative importance of the two deviations is provided by the error,

$$
\Delta \psi^{G}=\frac{h^{3}}{24}\left\{\frac{3}{2} e^{-2 L}+\left(\frac{h}{15}\right)^{2}\right\}^{1 / 2}+\ldots
$$


computed as the relative rms deviation from the continuum solution,

$$
(\Delta \psi)^{2} \equiv \frac{\left\langle\psi-\psi^{\text {cont }}, \psi-\psi^{\text {cont }}\right\rangle}{\left\langle\psi^{\text {cont }}, \psi^{\text {cont }}\right\rangle}
$$

We see that the unphysical component of the solution may be neglected, and that fourth-order accuracy is restored, if

$$
e^{-L}<\sqrt{\frac{2}{3}} \frac{h}{15}
$$

For a given $L$, this places a lower bound on the range of $h$ over which quartic convergence may be achieved. Nevertheless, the bound is not too stringent; for instance, if $L=5$, then this bound is $h>0.12$, while for $L=10$, we have $h>8 \times 10^{-4}$.

Negative fluxes in the outflow $G$ scheme will certainly occur if $r_{+}<0$, a condition that translates to zone widths $h>3$. However, this neglects the contribution that. the $\alpha_{-}$term makes to the full solution. Nevertheless, a detailed analysis including this term reveals that the condition $h>3$ is the necessary and sufficient condition for the occurence of negative fluxes.

The quadratic $(\mathrm{Q})$ scheme is also fourth-order accurate, with solutions of the form $\psi_{j}^{Q}=\psi(0) r_{+}^{j}$, where

$$
r_{+}=\frac{1-h / 2+h^{2} / 12}{1+h / 2+h^{2} / 12} .
$$

In the continuum limit, one finds

$$
\psi_{j}^{Q} \rightarrow \psi^{\text {cont }}(x)\left(1+\frac{x h^{4}}{720}+\mathcal{O}\left(h^{6}\right)\right) .
$$

From the known form of $\psi_{j}$, the quadratic component $\tilde{\psi}_{j}$ is easily constructed using Eq. (13). Besides being highly accurate, fluxes computed with quadratic scheme are never negative: the "hopping" parameter $r_{+}$is always positive, possessing a minimum value of $1 / 4$ when $h=3$.

The accuracy of the diamond-difference (DD), linear discontinuous (LD and MLD), outflow Galerkin $(G)$, and quadratic $(Q)$ schemes predicted above have been confirmed by numerical computations on a sample problem described by an absorber 5 mean free paths thick which experiences a unit incoming flux. The rms error $\Delta \psi$ in the absorber (computed using Eq. (26)) for each of the schemes is presented in Figure 1 over a range of zone widths, $10^{-2} \leq h \leq 2$. All computations were performed on an IBM RISC/System 6000 using 64 bit arithmetic. From Figure 1 it is clear that scaling behaviour of $\Delta \psi$ with respect to $h$ sets in at fairly large zone widths, e.g. for 
$h \sim 1$. Numerical experiments have also been performed confirming our predictions above regarding the onset of negative fluxes in the DD, LD and G scheme. These properties are summarized in Table 1.

In addition to accuracy, another important criterion for evaluating the difference schemes is computational cost. In Figure 2, a plot of accuracy versus computational time is presented for the same sample problem described above. To first approximation, the computational cost is proportional to $N \propto 1 / h$, and so the asymptotic slopes at large times (small $h$ ) of these log-log curves may also be used to determine the scheme accuracy.

It is evident from these Figures that the quadratic scheme is the superior scheme with respect to both accuracy and computational speed. Indeed, even for relatively large zone widths $h \sim 1$, the error in the $Q$ solution is nearly two orders of magnitude smaller than the error of the popular diamond-difference scheme. This property, plus the absence of negative fluxes, argues cogently for the quadratic scheme as the method of choice in solving the transport equation.

Figure 2 also shows that the outflow $\mathrm{G}$ scheme, while highly accurate, has larger time requirements than the other schemes. The reason for this is easily understood: The set of equations given by $(11), \psi_{0}=\psi(0)$ and (24) defines a linear system, $A \psi=\mathrm{b}$, where the matrix $A$ is banded, as opposed to the lower-triangular systems encountered in the other schemes we have considered. There is an additional cost in inverting this matrix. ${ }^{2}$ However, when we consider the case of nontrivial scattering and multiple ordinates in the next section, we shall see that this additional overhead becomes relatively unimportant.

\section{The case of uniform $\sigma_{T}, \sigma_{A}$ and $Q$}

In this section, we extend our comparison of finite-difference schemes for the transport equation (1) to the case of constant (but unequal) total and absorption cross sections, $\sigma_{T}$ and $\sigma_{A}$, and constant sources $Q$.

To simplify the notation somewhat, let us introduce the rescalings, $x=\sigma_{T} z, q=$ $Q / \sigma_{T}$ and $s=\sigma_{A} / \sigma_{T}$. We shall also adopt the familiar Gauss-Legendre quadratures to discretize the angular variable $\mu$. The quadrature points $\mu_{m}$ and weights $w_{m}$,

\footnotetext{
${ }^{2}$ In the computations presented in this paper, banded matrices were inverted using the subroutines DGBTRF and DGBTRS of the linear algebra package LAPACK, version 1.0 [21].
} 
$m=1, \ldots, M$, obey

$$
\begin{aligned}
\mu_{M+1-m} & =-\mu_{m}, \\
w_{M+1-m} & =w_{m}, \text { and } \\
\sum_{m=1}^{M} w_{m} & =2 .
\end{aligned}
$$

(We shall always choose $M$ to be even to avoid the singular point at $\mu=0$.) Then the equation to be solved is

$$
\mu_{m} \frac{d \psi_{m}(x)}{d x}+\psi_{m}(x)=(1-s) \phi(x)+q
$$

where $\psi_{m}(x) \equiv \psi\left(x, \mu_{m}\right)$ and the scalar flux $\phi(x)$ is given by

$$
\phi(x)=\frac{1}{2} \sum_{m=1}^{M} w_{m} \psi_{m}(x) .
$$

In any finite region, $x \in[0, L]$, a unique solution of this equation exists, provided the incoming flux,

$$
\begin{aligned}
& \psi_{m}(0) \text { for } \mu_{m}>0 \\
& \psi_{m}(L) \text { for } \mu_{m}<0
\end{aligned}
$$

is fully specified [1].

For our discussion below, it is useful to have at hand an analytical solution of Eq. (28). This is simple enough to do for $M=2$, for which $-\mu_{1}=\mu_{2}=1 / \sqrt{3}$ and $w_{1}=w_{2}=1$. Define $\lambda=\sqrt{3 s}$ and $b_{ \pm}=1 \pm \sqrt{s}$. Then the continuum solution for $M=2$ is given by

$$
\begin{aligned}
& \psi_{1}^{\text {cont }}(x)=\frac{q}{s}+a_{+} b_{-} e^{-\lambda x}+a_{-} b_{+} e^{\lambda x}, \\
& \psi_{2}^{\text {cont }}(x)=\frac{q}{s}+a_{+} b_{+} e^{-\lambda x}+a_{-} b_{-} e^{\lambda x},
\end{aligned}
$$

where the coefficients $a_{ \pm}$are determined by inverting the relation,

$$
\left(\begin{array}{cc}
b_{-} e^{-\lambda L} & b_{+} e^{\lambda L} \\
b_{+} & b_{-}
\end{array}\right)\left(\begin{array}{c}
a_{+} \\
a_{-}
\end{array}\right)=\left(\begin{array}{c}
\psi_{1}(L)-q / s \\
\psi_{2}(0)-q / s
\end{array}\right) .
$$

The development of finite difference schemes for Eq. (28) proceeds as in the previous section. We expand the functions $\psi_{m}(x)$ in terms of the teepee functions $\varphi_{j}(x)$, viz.

$$
\psi_{m}(x)=\sum_{j} \psi_{m, j} \varphi_{j}(x)
$$


for the diamond difference and Galerkin schemes, add to this quadratic elements for the quadratic scheme,

$$
\psi_{m}(x)=\sum_{j}\left(\psi_{m, j} \varphi_{j}(x)+\tilde{\psi}_{m, j} \tilde{\varphi}_{j}(x)\right)
$$

while for the linear discontinuous schemes, $\psi_{m}(x)$ is expanded in terms of the histogram and slope functions, $H_{j}(x)$ and $\tilde{H}_{j}(x)$ :

$$
\psi_{m}(x)=\sum_{j}\left(\psi_{m, j} H_{j}(x)+\tilde{\psi}_{m, j} \tilde{H}_{j}(x)\right)
$$

The DD equations result by projecting Eq. (28) with the histogram functions $H_{j}$, yielding

$$
\mu_{m}\left(\psi_{m, j}-\psi_{m, j-1}\right)+\frac{\Delta x_{j}}{2}\left(\psi_{m, j}+\psi_{m, j-1}\right)=\frac{\Delta x_{j}}{2}(1-s)\left(\phi_{j}+\phi_{j-1}\right)+\Delta x_{j} q
$$

where $\Delta x_{j}=x_{j}-x_{j-1}$ and

$$
\phi_{j}=\frac{1}{2} \sum_{m=1}^{M} w_{m} \psi_{m, j}
$$

The problem is fully determined with a specification of the inflow boundary conditions $\left(x_{0}=0, x_{N}=L\right)$,

$$
\begin{aligned}
\psi_{m, N} & =\psi\left(L, \mu_{m}<0\right), \quad m=1, \ldots, \frac{M}{2} \\
\psi_{m, 0} & =\psi\left(0, \mu_{m}>0\right), \quad m=\frac{M}{2}+1, \ldots, M
\end{aligned}
$$

In the linear discontinuous finite element method, we test Eq. (28) with the functions $v_{j} \in\left\{H_{j}, \tilde{H}_{j}\right\}$, modifying the naive projection with the replacement,

$$
\begin{aligned}
& \left\langle v_{j}, \mu_{m} \frac{d \psi_{m}}{d x}\right\rangle \\
\rightarrow & \left\langle v_{j}, \mu_{m} \frac{d \psi_{m}}{d x}\right\rangle+ \begin{cases}v_{j}\left(x_{j}^{-}\right) \mu_{m}\left[\psi_{m}\left(x_{j}^{+}\right)-\psi_{m}\left(x_{j}^{-}\right)\right], & \mu_{m}<0 \\
v_{j}\left(x_{j-1}^{+}\right) \mu_{m}\left[\psi_{m}\left(x_{j-1}^{+}\right)-\psi_{m}\left(x_{j-1}^{-}\right)\right], & \mu_{m}>0\end{cases}
\end{aligned}
$$

to impose inflow boundary conditions on each cell, $\left[x_{j-1}, x_{j}\right]$. One obtains,

$$
\begin{aligned}
& {\left[\Delta x_{j}\left(\begin{array}{ll}
1 & 0 \\
0 & \theta
\end{array}\right)+\mu_{m}\left(\begin{array}{cc}
-1 & 1 \\
-1 & -1
\end{array}\right)\right]\left(\begin{array}{c}
\psi_{m, j} \\
\tilde{\psi}_{m, j}
\end{array}\right)+\mu_{m}\left(\psi_{m, j+1}-\tilde{\psi}_{m, j+1}\right)\left(\begin{array}{l}
1 \\
1
\end{array}\right)} \\
& =\Delta x_{j}\left(\begin{array}{c}
(1-s) \phi_{j}+q \\
\theta(1-s) \tilde{\phi}_{j}
\end{array}\right)
\end{aligned}
$$


for $m=1, \ldots, M / 2$, and

$$
\begin{aligned}
& {\left[\Delta x_{j}\left(\begin{array}{cc}
1 & 0 \\
0 & \theta
\end{array}\right)+\mu_{m}\left(\begin{array}{cc}
1 & 1 \\
-1 & 1
\end{array}\right)\right]\left(\begin{array}{c}
\psi_{m, j} \\
\tilde{\psi}_{m, j}
\end{array}\right)+\mu_{m}\left(\psi_{m, j-1}+\tilde{\psi}_{m, j-1}\right)\left(\begin{array}{c}
-1 \\
1
\end{array}\right)} \\
& =\Delta x_{j}\left(\begin{array}{c}
(1-s) \phi_{j}+q \\
\theta(1-s) \tilde{\phi}_{j}
\end{array}\right)
\end{aligned}
$$

for $m=M / 2+1, \ldots, M$, where $\tilde{\phi}_{j}$ is defined analogously to $\phi_{j}$ in (29), replacing $\psi_{m, j}$ by $\tilde{\psi}_{m, j}$. The inflow boundary conditions for this scheme read

$$
\begin{aligned}
\psi_{m, N+1}-\tilde{\psi}_{m, N+1} & =\psi\left(L, \mu_{m}<0\right), \quad m=1, \ldots, \frac{M}{2}, \\
\psi_{m, 0}+\tilde{\psi}_{m, 0} & =\psi\left(0, \mu_{m}>0\right), \quad m=\frac{M}{2}+1, \ldots, M,
\end{aligned}
$$

which, together with the difference equations (34) and (35), completely determine the quantities, $\psi_{m, j}$ and $\tilde{\psi}_{m, j}$, for $j=1, \ldots, N$.

The Galerkin scheme results from testing (28) with the teepee functions $\varphi_{j}$, giving

$$
\begin{aligned}
& \frac{\mu_{m}}{2}\left(\psi_{m, j+1}-\psi_{m, j-1}\right) \\
+ & \frac{\Delta x_{j+1}}{6} \psi_{m, j+1}+\left(\frac{\Delta x_{j+1}}{3}+\frac{\Delta x_{j}}{3}\right) \psi_{m, j}+\frac{\Delta x_{j}}{6} \psi_{m, j-1} \\
= & (1-s)\left\{\frac{\Delta x_{j+1}}{6} \phi_{j+1}+\left(\frac{\Delta x_{j+1}}{3}+\frac{\Delta x_{j}}{3}\right) \phi_{j}+\frac{\Delta x_{j}}{6} \phi_{j-1}\right\} \\
+ & \frac{1}{2}\left(\Delta x_{j+1}+\Delta x_{j}\right) q
\end{aligned}
$$

for $m=1, \ldots, M$ and $j=1, \ldots, N-1$. In addition to the inflow boundary conditions (33), it is clear that we require $M$ two-point conditions to initialize the scheme. Taking our cue from the successful outflow initialization of the previous section, we impose the equations,

$$
\mu_{m}\left(\psi_{m, 1}-\psi_{m, 0}\right)+\frac{\Delta x_{1}}{2}\left(\psi_{m, 1}+\psi_{m, 0}\right)=\frac{\Delta x_{1}}{2}(1-s)\left(\phi_{1}+\phi_{0}\right)+\Delta x_{1} q
$$

for $m=1, \ldots, M / 2$, and

$$
\mu_{m}\left(\psi_{m, N}-\psi_{m, N-1}\right)+\frac{\Delta x_{N}}{2}\left(\psi_{m, N}+\psi_{m, N-1}\right)=\frac{\Delta x_{N}}{2}(1-s)\left(\phi_{N}+\phi_{N-1}\right)+\Delta x_{N} q,
$$

for $m=M / 2+1, \ldots, M$. We recognize (38) and (39) as the DD equations (32) evaluated at $j=1$ and $j=N$, respectively. 
Finally, the quadratic scheme is produced by testing with the histogram and slope functions, $H_{j}(x)$ and $\tilde{H}_{j}(x)$, in every zone, resulting in the following extension of the diamond-difference equations,

$$
\begin{aligned}
& \psi_{m, j}-\psi_{m, j-1}+\frac{\Delta x_{j}}{2 \mu_{m}}\left(\psi_{m, j}+\psi_{m, j-1}\right)+\frac{1}{12}\left(\frac{\Delta x_{j}}{\mu_{m}}\right)^{2}\left(\psi_{m, j}-\psi_{m, j-1}\right) \\
= & (1-s)\left(\frac{\Delta x_{j}}{2 \mu_{m}}\left(\phi_{j}+\phi_{j-1}\right)+\frac{1}{12}\left(\frac{\Delta x_{j}}{\mu_{m}}\right)^{2}\left(\phi_{m, j}-\phi_{m, j-1}\right)+\frac{2 \Delta x_{j}}{3 \mu_{m}} \tilde{\phi}_{j}\right) \\
+ & \frac{\Delta x_{j}}{\mu_{m}} q
\end{aligned}
$$

and

$$
\tilde{\psi}_{m, j}=\frac{\Delta x_{j}}{8 \mu_{m}}\left(\psi_{m, j}-\psi_{m, j-1}+(1-s)\left(\phi_{j}-\phi_{j-1}\right)\right)
$$

where

$$
\tilde{\phi}_{j}=\frac{1}{2} \sum_{m} w_{m} \tilde{\psi}_{m, j}=\frac{\Delta x_{j}}{16} \sum_{m} \frac{w_{m}}{\mu_{m}}\left(\psi_{m, j}-\psi_{m, j-1}\right) .
$$

The boundary conditions are again supplied by Eq. (33).

For the case of two ordinates $(M=2)$ and uniform grid spacing $\left(\Delta x_{j}=h\right)$, the discrete difference equations given above are sufficiently tractable to be solved analytically using the methods employed in section $2 .^{3}$ First note that the nonhomogeneous source terms may be eliminated by the translation,

$$
\psi_{m, j}=\frac{q}{s}+\psi_{m, j}^{\text {homo }} .
$$

We then seek solutions of the homogeneous equation by making the Ansatz,

$$
\psi_{m, j}^{h o m o}=\Psi_{m} r^{j},
$$

develop secular equations for $r$, and solve them for $r$ and the corresponding eigenvectors $\Psi^{(r)}$. The expressions so obtained for the components $\Psi_{m}^{(r)}$ are rather cumbersome and are omitted here for the sake of brevity.

The secular equation obtained by inserting (42) and (43) in the diamond difference equations (32) is

$$
s-\left[\frac{2}{\sqrt{3} h} \frac{1-r}{1+r}\right]^{2}=0,
$$

whose solutions are $r=r_{ \pm}$, where

$$
r_{+}=\frac{1}{r_{-}}=\frac{1-h \lambda / 2}{1+h \lambda / 2}
$$

\footnotetext{
${ }^{3}$ Note that the definition, $h=\sigma_{T} \Delta z$, differs from that of the previous section by a factor of $1 /|\mu|$.
} 
(compare to Eq. (15)). Working out the corresponding eigenvectors $\Psi_{m}^{( \pm)}$yields the general solution,

$$
\begin{aligned}
& \psi_{1, j}^{D D}=\frac{q}{s}+\alpha_{+} b_{-} r_{+}^{j}+\alpha_{-} b_{+} \frac{1}{r_{+}^{j}}, \\
& \psi_{2, j}^{D D}=\frac{q}{s}+\alpha_{+} b_{+} r_{+}^{j}+\alpha_{-} b_{-} \frac{1}{r_{+}^{j}},
\end{aligned}
$$

where $\alpha_{ \pm}$are fixed by the boundary conditions (33). There is an obvious correspondence with the continuum solution (30), given by $r_{+}^{j} \leftrightarrow e^{-\lambda x}$ and $\alpha_{ \pm} \leftrightarrow a_{ \pm}$. Indeed, the coefficients $\alpha_{ \pm}$obey a matrix equation analogous to Eq. (31) obtained by replacing $e^{-\lambda L}$ by $r_{+}^{N}$. Noting that

$$
r_{+}^{j} \rightarrow e^{-\lambda x}\left(1-\frac{\lambda^{3} h^{2} x}{12}+\mathcal{O}\left(h^{4}\right)\right)
$$

as $h \rightarrow 0$, it is evident that in the continuum limit the DD solution differs from the exact solution (30) by terms of order $h^{2}$. This is most simply illustrated in the dual limits of small zones and thick media,

$$
\text { Continuum limit: } \quad h \rightarrow 0,
$$

$$
\text { Thick medium limit: } \quad \lambda L>>1 \text {. }
$$

In this case, the $\mathcal{L}^{2}$ deviation of the scalar flux $\phi^{D D}(x)$ from the $\mathrm{M}=2$ continuum scalar flux $\phi^{\text {cont }}(x)$, given by Eqs. (29) and (30), is

$$
\frac{1}{L} \int_{0}^{L} d x\left(\phi^{D D}(x)-\phi^{c o n t}(x)\right)^{2}=\left(\frac{\lambda^{2} h^{2}}{24}\right)^{2} \frac{\vec{a}_{+}^{2}+\bar{a}_{-}^{2}}{\lambda L}+\ldots
$$

where

$$
\bar{a}_{+}=\frac{1}{b_{+}}\left(\psi_{2}(0)-\frac{q}{s}\right) \text { and } \bar{a}_{-}=\frac{1}{b_{+}}\left(\psi_{1}(L)-\frac{q}{s}\right),
$$

implying an error of $\Delta \phi^{D D} \sim h^{2}$, as expected.

The secular equation for the linear discontinuous schemes, Eqs. (34) and (35), is

$$
c_{+}(1+r)^{2}-c_{-}(1-r)^{2}=0,
$$

where

$$
\begin{aligned}
& c_{+}=\lambda^{2} h^{2}\left(1+\frac{\theta \lambda h}{2 \sqrt{s}}\right)\left(1+\frac{\theta \sqrt{s} \lambda h}{2}\right), \\
& c_{-}=\left(2+\frac{\theta \lambda h}{\sqrt{s}}+\frac{\theta \lambda^{2} h^{2}}{2}\right)\left(2+\theta \sqrt{s} \lambda h+\frac{\theta \lambda^{2} h^{2}}{2}\right) .
\end{aligned}
$$


As in the DD case, the two roots $r_{ \pm}$of this equation are inversely related and are given by

$$
r_{+}=\frac{1}{r_{-}}=\frac{\sqrt{c_{-}}-\sqrt{c_{+}}}{\sqrt{c_{-}}+\sqrt{c_{+}}} .
$$

In the continuum limit, one finds,

$$
r_{+}^{j} \rightarrow e^{-\lambda x}\left(1+\left(\theta-\frac{1}{3}\right) \frac{\lambda^{3} h^{2} x}{4}-\left(\sqrt{s}+\frac{1}{\sqrt{s}}\right) \theta^{2} \frac{\lambda^{4} h^{3} x}{16}+\mathcal{O}\left(h^{4}\right)\right),
$$

promising third-order accuracy if $\theta=1 / 3$, second-order accuracy otherwise. Whether or not this degree of accuracy is realized depends in detail on the continuum-limit behaviour of the coefficients appearing in the full solution,

$$
\begin{aligned}
& \psi_{1, j}^{(M) L D}=\frac{q}{s}+\alpha_{+}\left(\Psi_{1}^{(+)}-\tilde{\Psi}_{1}^{(+)}\right) r_{+}^{j+1}+\alpha_{-}\left(\Psi_{1}^{(-)}-\tilde{\Psi}_{1}^{(-)}\right) \frac{1}{r_{+}^{j+1}} \\
& \psi_{2, j}^{(M) L D}=\frac{q}{s}+\alpha_{+}\left(\Psi_{2}^{(+)}+\tilde{\Psi}_{2}^{(+)}\right) r_{+}^{j}+\alpha_{-}\left(\Psi_{2}^{(-)}+\tilde{\Psi}_{2}^{(-)}\right) \frac{1}{r_{+}^{j}}
\end{aligned}
$$

Here, as before, the coefficients $\alpha_{ \pm}$are fixed by the boundary conditions (36). The other quantities, $\Psi_{m}^{( \pm)}$and $\tilde{\Psi}_{m}^{( \pm)}$, are eigenvector components corresponding to the two roots $r_{ \pm}$. We have chosen a normalization for these components such that, as $h \rightarrow 0$,

$$
\begin{aligned}
& \Psi_{1}^{(+)}-\tilde{\Psi}_{1}^{(+)}=\Psi_{2}^{(-)}+\tilde{\Psi}_{2}^{(-)} \rightarrow b_{-} \\
& \Psi_{1}^{(-)}-\tilde{\Psi}_{1}^{(-)}=\Psi_{2}^{(+)}+\tilde{\Psi}_{2}^{(+)} \rightarrow b_{+}
\end{aligned}
$$

The error of the solution produced by this scheme may be examined in the dual limits (45). One finds

$$
\begin{aligned}
& \frac{1}{L} \int_{0}^{L} d x\left(\phi^{(M) L D}(x)-\phi^{c o n t}(x)\right)^{2} \\
= & \left\{\left(\left(\theta-\frac{1}{3}\right) \frac{\lambda^{2} h^{2}}{8}-\left(1+\frac{1}{\sqrt{s}}\right) \frac{\theta^{2} \lambda^{3} h^{3}}{32}\right)^{2}+\left(\frac{\theta^{2} \lambda^{3} h^{3} b_{-}}{32}\right)^{2}\right\} \frac{\vec{a}_{+}^{2}+\bar{a}_{-}^{2}}{\lambda L}+\ldots
\end{aligned}
$$

verifying third-order accuracy for $\theta=1 / 3$.

A similar exercise can be conducted for the Galerkin scheme, Eqs. (37) - (39). The general solution is of the form,

$$
\begin{aligned}
& \psi_{1, j}^{G}=\frac{q}{s}+b_{-}\left(\alpha_{+} r_{+}^{j}+\beta_{+} r_{-}^{j}\right)+b_{+}\left(\frac{\alpha_{-}}{r_{+}^{j}}+\frac{\beta_{-}}{r_{-}^{j}}\right), \\
& \psi_{2, j}^{G}=\frac{q}{s}+b_{+}\left(\alpha_{+} r_{+}^{j}+\beta_{+} r_{-}^{j}\right)+b_{-}\left(\frac{\alpha_{-}}{r_{+}^{j}}+\frac{\beta_{-}}{r_{-}^{j}}\right),
\end{aligned}
$$


where there are now four constants, $\alpha_{ \pm}$and $\beta_{ \pm}$to be determined. These are fixed by the two boundary conditions (33) and the two DD outflow equations, Eqs. (38) and (39). The roots $r_{ \pm}$are given by

$$
r_{ \pm}=\frac{-2 \lambda h / 3 \pm \sqrt{1+\lambda^{2} h^{2} / 3}}{1+\lambda h / 3}
$$

yielding in the limit $h \rightarrow 0$,

$$
\begin{aligned}
r_{+}^{j} & \rightarrow e^{-\lambda x}\left(1-\frac{\lambda^{5} h^{4} x}{180}+\mathcal{O}\left(h^{6}\right)\right), \\
\left|r_{-}\right|^{j} & \rightarrow e^{\lambda x / 3}\left(1-\frac{2 \lambda^{3} h^{2} x}{81}+\mathcal{O}\left(h^{4}\right)\right) .
\end{aligned}
$$

We recognize, once again, the emergence of nonphysical, oscillating (note $\left.r_{-}<0\right)$ components in the solution. However, unlike the case of the pure absorber considered in section 2 , these components are not exponentially suppressed by initializing the outflowing flux. Indeed, in the continuum/thick medium limit, one finds

$$
\frac{1}{L} \int_{0}^{L} d x\left(\phi^{G}(x)-\phi^{\text {cont }}(x)\right)^{2}=\left(\frac{\lambda^{3} h^{3}}{24}\right)^{2}\left(\frac{b_{-}}{b_{+}}\right)^{2}\left\{\left(\frac{b_{-}}{b_{+}}\right)^{2}+3\right\} \frac{\bar{a}_{+}^{2}+\bar{a}_{-}^{2}}{2 \lambda L}+\ldots
$$

representing only third-order accuracy. Notice that these leading terms vanish in the pure absorber limit, $s=\sigma_{A} / \sigma_{T} \rightarrow 1\left(b_{-} \rightarrow 0\right)$, and it is only in this limit that fourth-order accuracy is regained.

As expected, the secular equation for the quadratic scheme is an extension of what was encountered in the diamond difference scheme:

$$
s-\left[\frac{2}{\sqrt{3} h}\left(1+\frac{s h^{2}}{4}\right) \frac{1-r}{1+r}\right]^{2}=0 .
$$

This has solutions $r=r_{ \pm}$given by

$$
r_{+}=\frac{1}{r_{-}}=\frac{1-h \lambda / 2+h^{2} \lambda^{2} / 12}{1+h \lambda / 2+h^{2} \lambda^{2} / 12}
$$

possessing the continuum limit

$$
r_{+}^{j} \rightarrow e^{-\lambda x}\left(1+\frac{\lambda^{5} h^{4} x}{720}+\mathcal{O}\left(h^{6}\right)\right)
$$

The discrete solution $\psi_{m, j}^{Q}$ has precisely the same form (44) as in the DD case, where the coefficients $\alpha_{ \pm}$are now fixed to higher order in $\lambda h$ (but which we forgo giving 
here due to their length). Examining the solution in the dual limit (45), one confirms the expected fourth-order accuracy:

$$
\frac{1}{L} \int_{0}^{L} d x\left(\phi^{Q}(x)-\phi^{\text {cont }}(x)\right)^{2}=\left(\frac{\lambda^{4} h^{4}}{1440}\right)^{2} \frac{\bar{a}_{+}^{2}+\bar{a}_{-}^{2}}{\lambda L}+\ldots
$$

In the case of two ordinates, we have also determined the minimal condition on the zone width $h$ required to ensure that fluxes are positive, with results that are presented in Table 1. Here the relevant scaled zone width is $H=\lambda h=\sqrt{\sigma_{A} \sigma_{T}} \Delta z /|\mu|$, e.g. the projected zone width measured in units of the diffusion length $l_{D}=1 / \sqrt{\sigma_{A} \sigma_{T}}$. The parallels to the pure absorber case are evident from Table 1 . One notable difference is found for the LD scheme, where the onset of negative fluxes decreases from $H=3$ to $\sqrt{6}$ as $s=\sigma_{A} / \sigma_{T}$ decreases from 1 (pure absorption) to 0 (purely scattering).

The theoretical analysis given above has involved only two ordinates, and it is therefore of interest to test its conclusions with numerical experiments. For this purpose, we have computed the flux density on a test problem for two sets of ordinates: $M=2$ and 8 . Our test problem is specified by the set,

$$
\begin{aligned}
& q=s=\frac{1}{4} \\
& L=5 \\
& \psi(L, \mu<0)=\psi(0, \mu>0)=0
\end{aligned}
$$

For this choice of parameters, $\exp (-\lambda L)=0.013$ and so we are justified in neglecting exponential corrections, as the analysis above requires. The results are presented in Figures 3 - 6. The quantity denoted as the relative error $\Delta \phi$ in these Figures is defined, for a fixed quadrature, by the expression

$$
(\Delta \phi)^{2} \equiv \frac{\left\langle\phi-\phi^{\text {cont }}, \phi-\phi^{\text {cont }}\right\rangle}{\left\langle\phi^{\text {cont }}, \phi^{\text {cont }}\right\rangle} \text {. }
$$

First consider the case of two ordinates. As seen from Fig. 3, the $Q$ scheme is more accurate than the DD scheme by nearly two orders of magnitude for zone widths less than or equal to a mean free path. Furthermore, for $h<1$, a fit to the slopes gives the relative error of the DD and MLD solutions scaling like $h^{2}$, the LD and $\mathrm{G}$ solutions like $h^{3}$, and the $Q$ solution as $h^{4}$, as expected. Thus, with the exception of the $\mathrm{G}$ scheme, our results replicate what we found in the case of the pure absorber. The same may be said of the relative computational speed of each scheme, as displayed in Fig. 4, save that the overhead we observed in the pure absorber case when implementing outflow initialization is no longer significant. 
The same story is repeated in the case of 8 ordinates (Figs. 5 and 6). One qualitative difference is that for zone widths $h \geq 1$; all schemes given roughly comparable performance in both accuracy and speed. In particular, the $Q$ scheme emerges as the preferred scheme only when zone widths are less than a mean free path. Another feature of Fig. 6 worth noting is that the LD scheme outperforms the DD scheme only when $\Delta \phi<5 \times 10^{-4}$, corresponding to $h<0.2$ or $H<0.17$, while the MLD and Galerkin schemes are never competitive with the DD scheme in the range of zone widths considered.

\section{Summary and conclusions}

In the effort to obtain greater accuracy in solving the transport equation, we have examined several known, but apparently neglected, finite difference schemes - the Galerkin and quadratic schemes - based on expansions in linear and quadratic, piecewise continuous elements. In the simple setting of slab geometry with constant cross sections and sources, we have compared these schemes with the standard diamond difference scheme and with currently popular schemes using linear discontinuous elements.

The comparison does not reflect well on the Galerkin scheme as a method for integrating the transport equation: First, being a three-point scheme, we have shown how the $G$ scheme gives rise to an unphysical, oscillating component which spoils the potential accuracy of the scheme and easily leads to negative fluxes. The latter problem may be overcome in optically thick media by imposing two-point conditions on fluxes at the outflow boundaries. Nevertheless, in the general case with nonzero scattering, this tactic also produces solutions exhibiting only third-order accuracy, and the naive fourth-order accuracy of the Galerkin equations is lost. Third-order accuracy is an improvement over the diamond difference scheme, but this casual assessment overlooks the fact that the Galerkin scheme with outflow initialization is computationally more expensive to execute.

On the contrary, our investigation has indicated the superiority the quadratic scheme. It is fourth-order accurate and is only marginally more involved than the diamond difference scheme to implement. Furthermore, it eschews negative fluxes and, for zone widths less than a mean free path, is competitive with the DD scheme.

Our work raises the question whether finite elements of higher order polynomials (cubic, quartic, etc.) would result in even more accurate and efficient schemes. Results 
from a work by Martin and Duderstadt [11] using cubic elements do not support this expectation, although their study on this point was not thorough enough to permit a definite conclusion.

It remains for future work to determine whether or not this scorecard of finite difference methods for the-transport equation survives more complex geometries, nonuniform sources or material densities, or generalization to higher dimensions. Also, the question remains whether the Galerkin and quadratic schemes, based as they are on continuous finite elements, can accomodate sharp material boundaries as successfully as the linear discontinuous schemes.

We are grateful for discussions with Alan Hindmarsh. This work was supported by the Lawrence Livermore National Laboratory under the LDRD Project No. 92SR-009 and by the Department of Energy under Contract No. W-7405-Eng-48. 


\section{References}

[1] K.M. Case and P.F. Zweifel, Linear Transport Theory (Addison - Wesley, Reading, Mass., 1967).

[2] B.G. Carlson and K.D. Lathrop, in Computing Methods in Reactor Physics, ed. by H. Greenspan, C.N. Kelber and D. Okrent (Gordon and Breach, New York, 1968), Ch. 3.

[3] E.E. Lewis and W.F. Miller, Jr., Computational Methods of Neutron Transport (Wiley-Interscience, New York, 1984).

[4] E.W. Larsen, J.E. Morel and W.F. Miller, Jr., J. Comp. Phys. 69, 283 (1987).

[5] E.W. Larsen and J.E. Morel, J. Comp. Phys. 83, 212 (1989); corrigendum, J. Comp. Phys. 91, 246 (1990).

[6] T.R. Hill, ONETRAN, A discrete ordinates finite element code for the solution of the one-dimensional, multigroup transport equation, LA-5990-MS, Los Alamos National Laboratory, 1973.

[7] R.H. Szilard and G.C. Pomraning, Trans. Theo. and Stat. Phys. 18, 255 (1989).

[8] M.L. Adams, Discontinuous finite-element transport solutions in the thick diffusion limit in Cartesian geometry, Proc. ANS Topical Meeting, Advances in Mathematics, Computations, and Reactor Physics, April 29 - May 2, 1991, Pittsburgh, PA, Vol. 5, Ch. 21.1, pp. 3-1 to 3-15.

[9] C. Börgers, E.W. Larsen and M.L. Adams, J. Comp. Phys. 98, 285 (1992).

[10] See, for example, G. Strang and G.J. Fix, An Analysis of the Finite Element Method (Prentice-Hall, Englewood Cliffs, New Jersey, 1973), Ch.1.

[11] W.R. Martin and J.J. Duderstadt, Nucl. Sci. Eng. 62, 371 (1977).

[12] R.E. Alcouffe, E.W. Larsen, W.F. Miller, Jr. and B.R. Wienke, Nucl. Sci. Eng. 71,111 (1979).

[13] S.M. Lee and R. Vaidyanathan, Nucl. Sci. Eng. 76, 1 (1979).

[14] E.W. Larsen and W.F. Miller, Jr., Nucl. Sci. Eng. 73, 76 (1980). 
[15] D.V. Gopinath, A. Natarajan and V. Sundararaman, Nucl. Sci. Eng. 75, 181 (1980).

[16] E.W. Larsen and P. Nelson, SIAM J. Numer. Anal. 19, 334 (1982).

[17] R. Vaidyanathan, Atomkernenergie 29, 301 (1977); Nucl. Sci. Eng. 72, 46 (1979).

[18] K.D. Lathrop, J. Comp. Phys. 4, 475 (1969).

[19] J.M. Ferguson, in Transactions of the American Nuclear Society 33, 328 (1979).

[20] A. Greenbaum and J.M. Ferguson, J. Comp. Phys. 64, 97 (1986).

[21] E. Anderson et al., LAPACK Users' Guide (SIAM, Philadelphia, 1992). 


\begin{tabular}{|l|cc|cc|}
\hline Scheme & \multicolumn{2}{|c|}{ Accuracy } & \multicolumn{2}{c|}{ Positivity condition } \\
& $\sigma_{S}=0$ & $\sigma_{S} \neq 0$ & $\sigma_{S}=0$ & $\sigma_{S} \neq 0$ \\
\hline $\mathrm{DD}$ & 2 & 2 & $h<2$ & $H<2$ \\
$\mathrm{LD}$ & 3 & 3 & $h<3$ & $H<\sqrt{6}\left(\sigma_{S}=\sigma_{T}\right)$ \\
$\mathrm{MLD}$ & 2 & 2 & None & None \\
$\mathrm{G}$ & 4 & 3 & $h<3$ & $H<3$ \\
$\mathrm{Q}$ & 4 & 4 & None & None \\
\hline
\end{tabular}

Table 1

\section{Table Captions}

Table I: Accuracy and minimal conditions for positivity (e.g. $r_{+}>0$ ) in five finitedifference schemes for the linear transport equation, as determined by exact analysis. Two cases are considered: (1) a purely absorbing medium $\left(\sigma_{S}=0\right)$ and $(2)$ a medium with uniform scattering $\left(\sigma_{S} \neq 0\right)$. In the second case, angles are limited to 2 Gauss-Legendre ordinates. The scaled zone widths $h$ and $H$ are related to the physical zone widths $\Delta z$ via $h=\sigma_{T} \Delta z /|\mu|$ and $H=\sqrt{s} h=\sqrt{\sigma_{T} \sigma_{A}} \Delta z /|\mu|$. 


\section{Figure Captions}

Fig. 1: Accuracy versus zone width $h=\sigma_{T} \Delta z /|\mu|$ of solutions determined by Eqs. (8), (10), (11), and (13), as applied to a pure absorber of width 5.0 in dimensionless units. Presented are results using the diamond difference scheme (DD), the linear discontinuous scheme for $\theta=1 / 3$ (LD) and $\theta=1$ (MLD), the Galerkin scheme (G), and the quadratic scheme (Q).

Fig. 2: Accuracy versus computational time (in seconds) of the DD, LD, MLD, G and $\mathrm{Q}$ solutions for a pure absorber of width 5.0 in dimensionless units.

Fig. 3: Accuracy of the DD, LD, MLD, G, and $Q$ solutions as a function of zone width $h=\sigma_{T} \Delta z$ for the scattering and absorbing medium defined by (48). The angular variable $\mu$ is discretized in $S_{2}$ Gauss-Legendre quadratures.

Fig. 4: Accuracy versus computational time (in seconds) of the DD, LD, MLD, G, and $\mathrm{Q}$ solutions for the scattering and absorbing medium defined by (48). The angular variable $\mu$ is discretized in $S_{2}$ Gauss-Legendre quadratures.

Fig. 5: $\quad$ Same as Fig. 3 using $S_{8}$ quadratures.

Fig. 6: Same as Fig. 4 using $S_{8}$ quadratures. 
Figure 1. Accuracy versus zone width

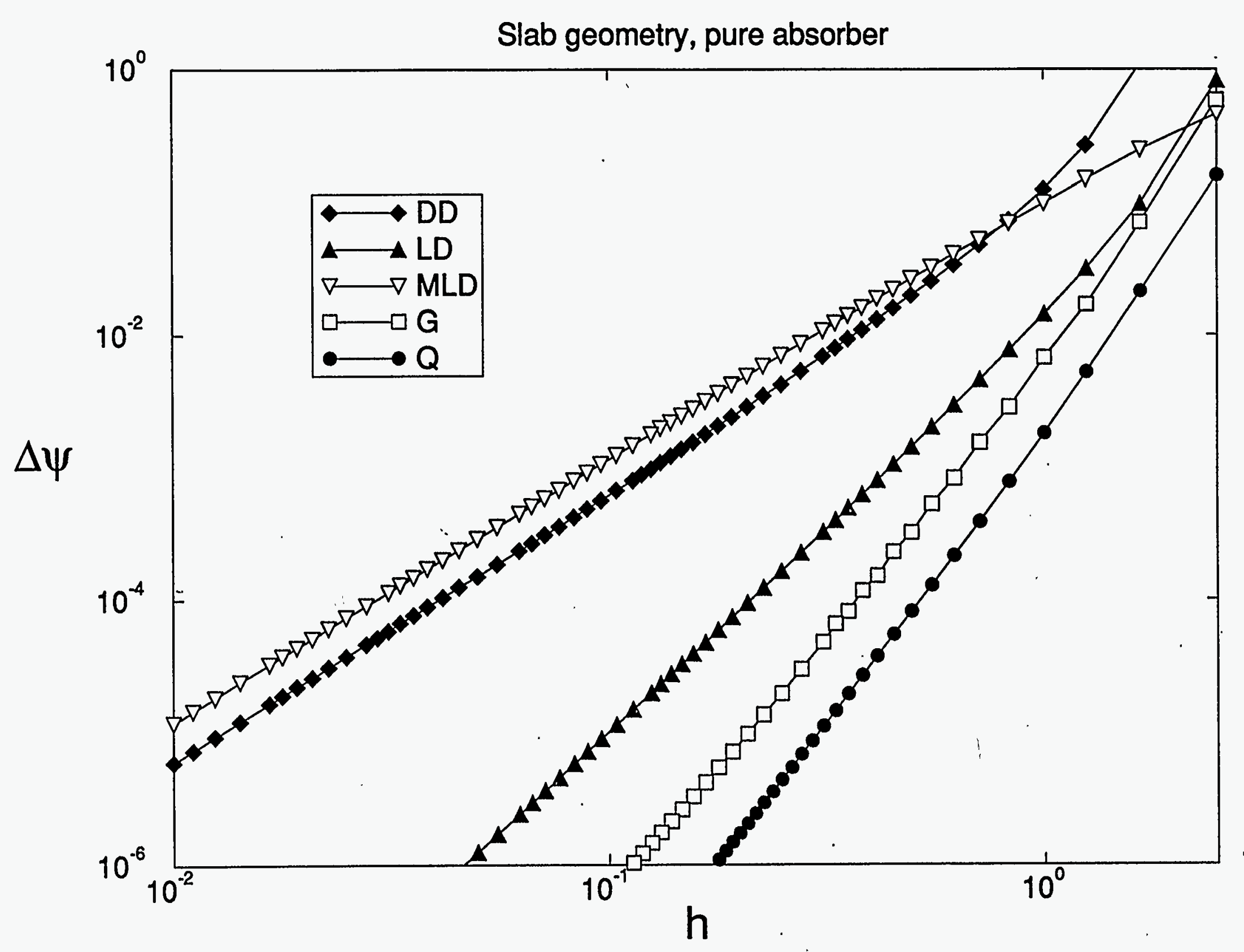




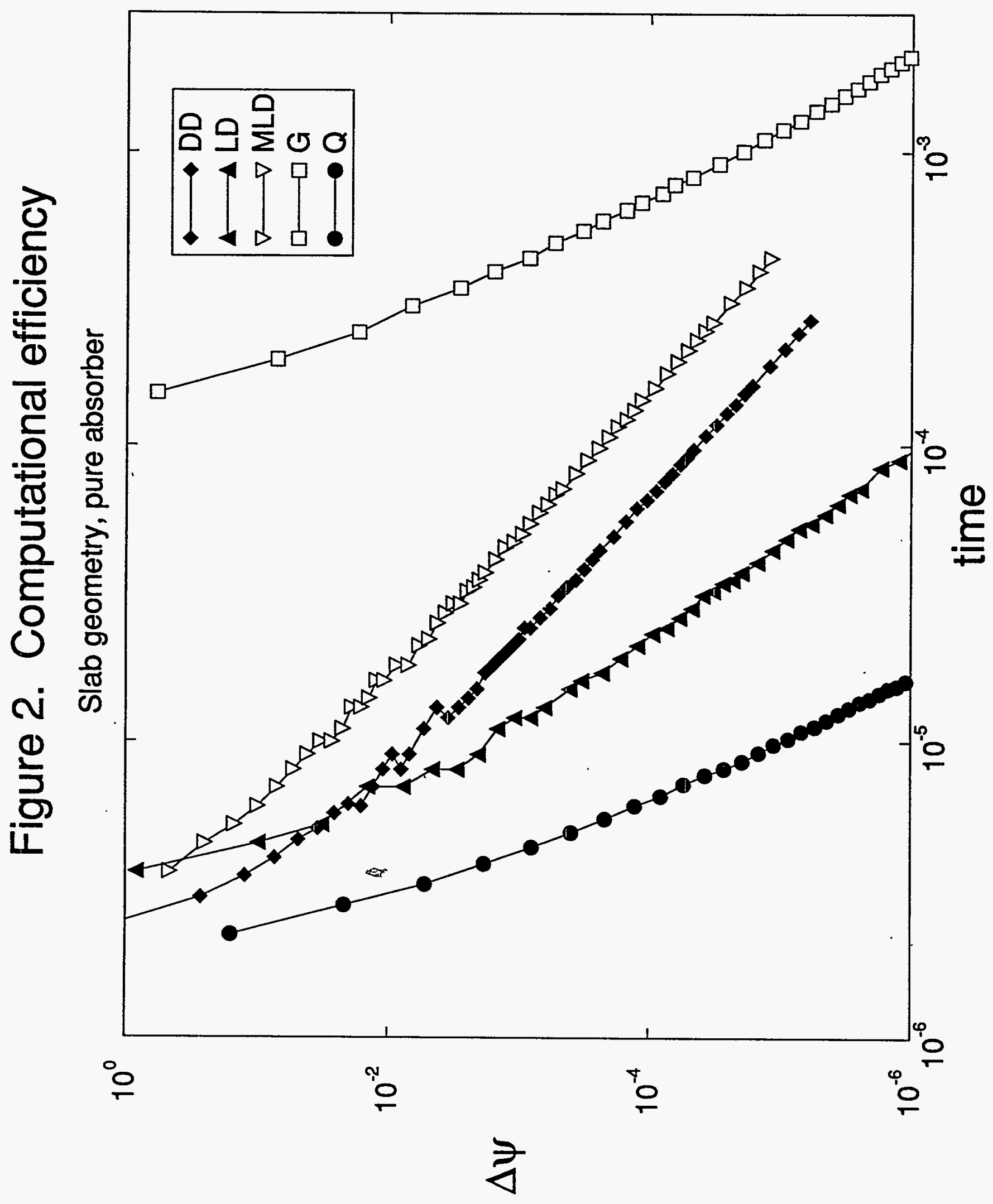




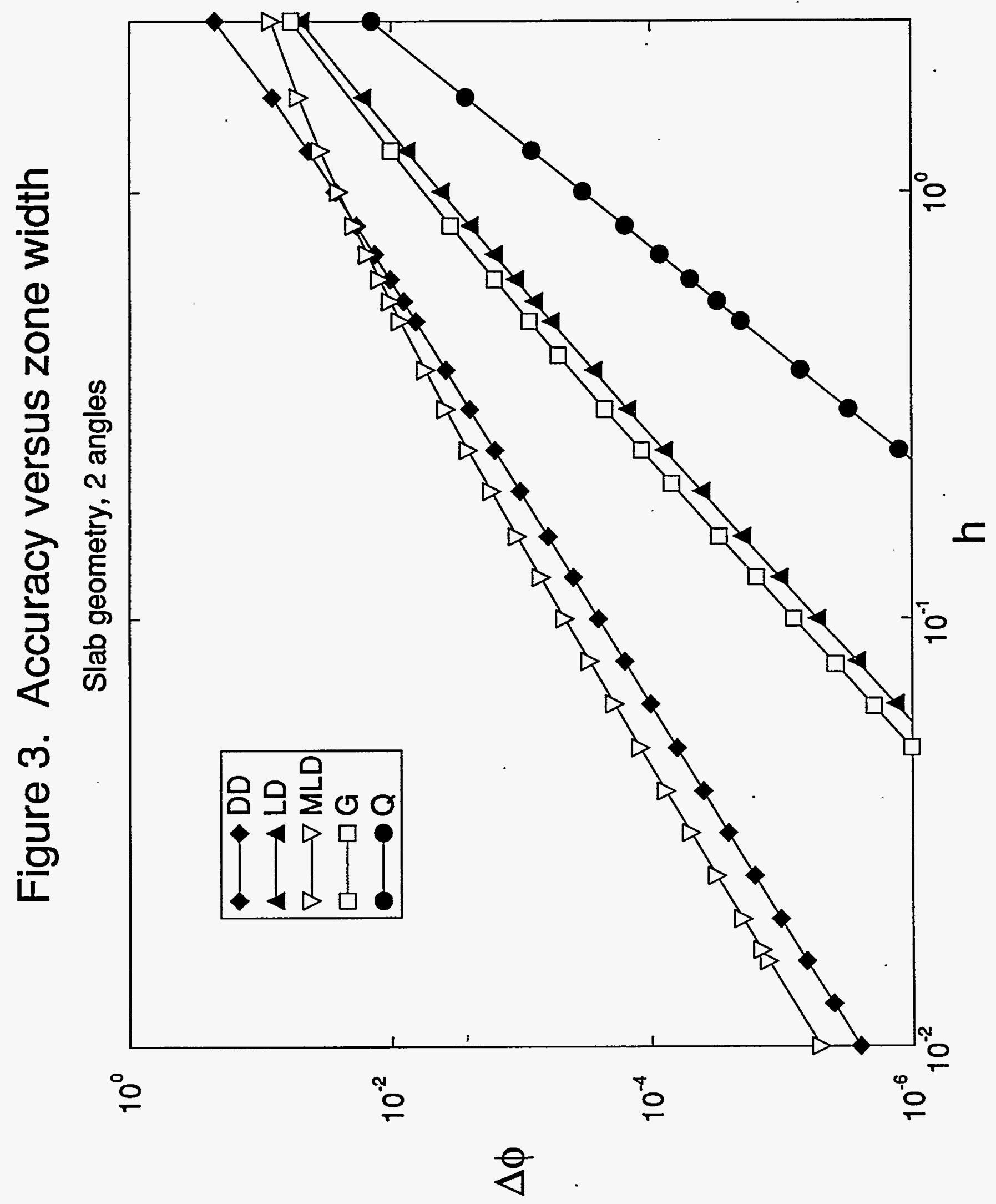




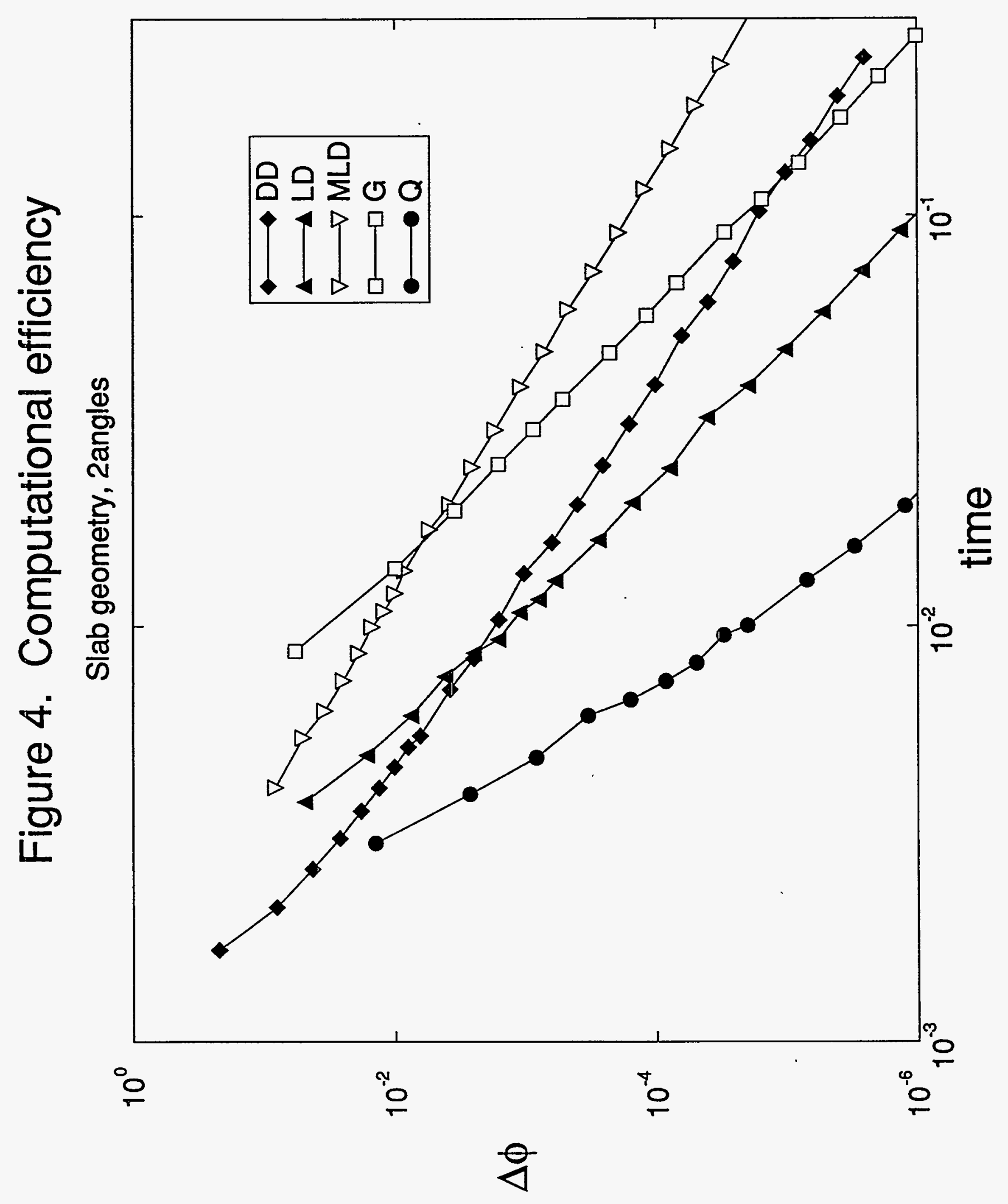




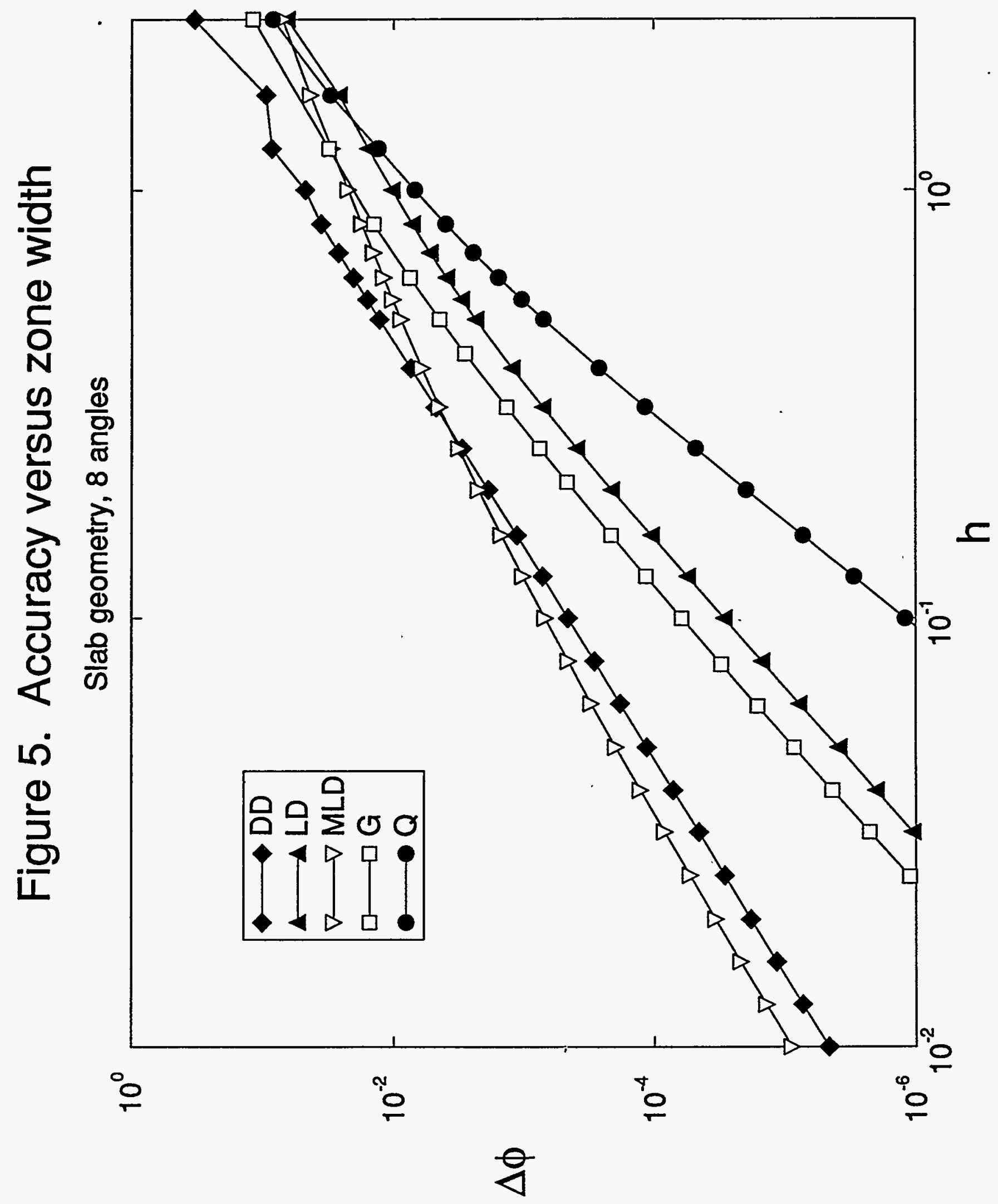




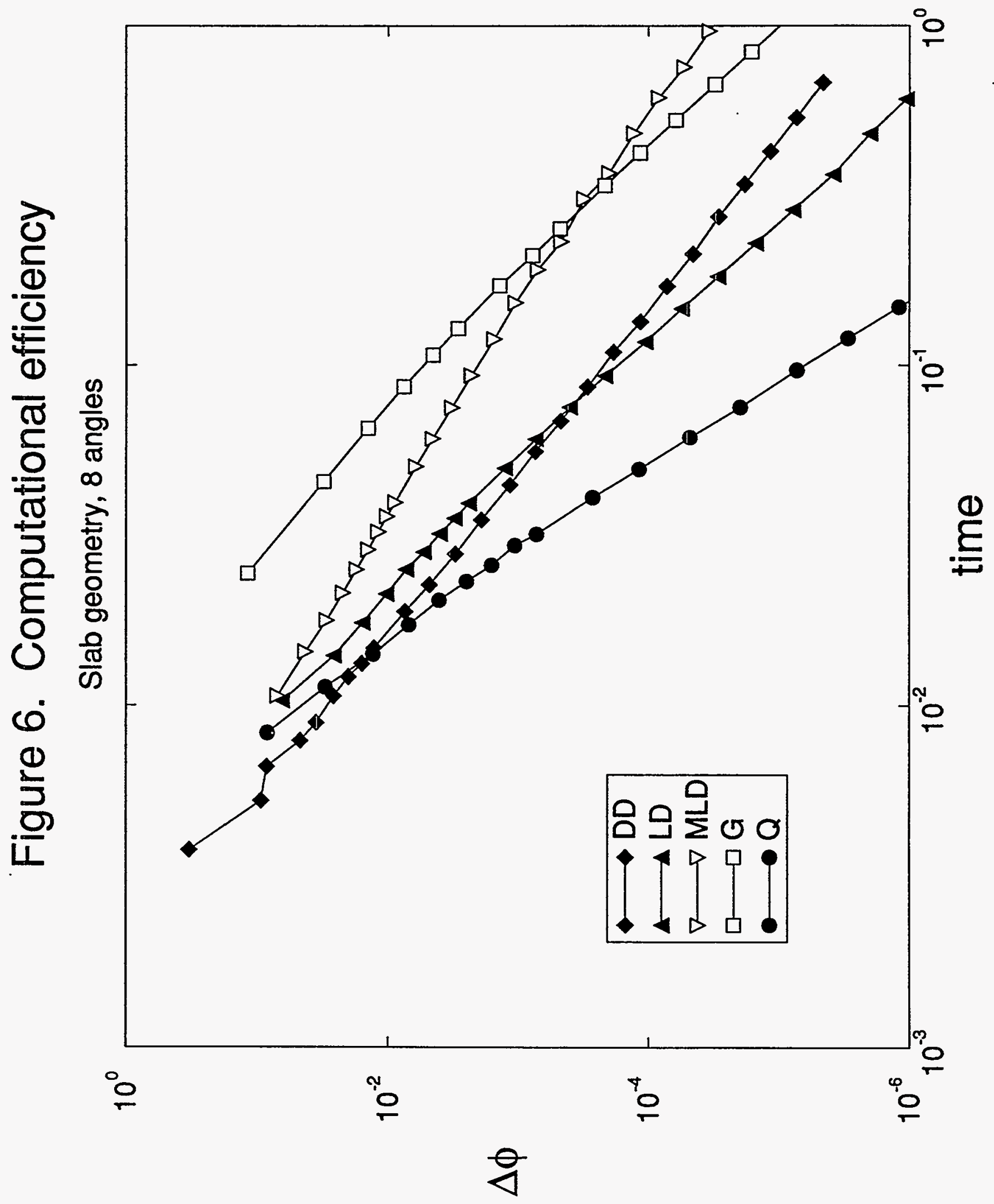

